\title{
La agenda noticiosa de la prensa escrita y las iglesias en México
}

\section{Sergio Inestrosa González ${ }^{1}$}

\begin{abstract}
A
LO LARGO DE ESTE REPORTE de investigación se pretende presentar una primera aproximación a la relación entre las distintas iglesias acreditadas en nuestro país y la agenda que la prensa escrita estableció durante el año de 1998.

El trabajo de investigación denominado "Cultura religiosa y medios de comunicación en México" fue desarrollado durante el presente año por un pequeño equipo de investigadores del Departamento de Comunicación de la Universidad Iberoamericana. Entre los objetivos del proyecto estuvo el revisar la agenda que la prensa escrita fue planteando a lo largo del año respecto a los acontecimientos de carácter religioso en nuestro país y en el ámbito internacional. Para cumplir con esta meta se hicieron dos exploraciones en seis periódicos de circulación nacional. ${ }^{2}$ La primera de las exploraciones se hizo entre febrero y mayo y la segunda de agosto a septiembre de 1998.

Sobre la base de esta doble recopilación de noticias se articuló el presente informe, el cual nos permite hacer una primera valoración respecto a cuatro hipótesis de trabajo que fueron planteadas desde un principio para guiar el trabajo de campo.

La primera de las hipótesis planteadas, conjetura sobre el hecho de que los medios de comunicación se ocupan de los asuntos religiosos (como del resto del acontecer noticioso), como si fueran asuntos que deben ser permanentemente renovados y que aparecen y desaparecen de la agenda atendiendo a su peso coyuntural.
\end{abstract}

La segunda hipótesis busca explorar la idea de que los medios no tienen, ni les interesa tener, expertos que se encarguen de dar un tratamiento serio a los

1. Universidad Iberoamericana.

2. Los periódicos consultados fueron: La Jornada, Excélsior, La Crónica, Reforma, El Financiero y El Universal. 
asuntos religiosos, como sí lo tienen en otros campos como la economía y la política.

En tercer lugar, se plantea la hipótesis de que los medios, al igual que ocurre con otros aspectos del acontecer noticioso, se ocupan de lo religioso atendiendo principalmente a poner de manifiesto las opiniones de los jerarcas de las iglesias y no de la feligresía.

Por último, constatamos que la mayoría de noticias estuvieron relacionadas directa y fundamentalmente con la Iglesia católica en detrimento de otros grupos religiosos que rara vez aparecen agendados.

El fondo del entramado teórico de este reporte está dado por la apuesta de distanciarse de la idea generalizada, entre muchos de nosotros, de que los medios de comunicación son instancias que transfieren información a sus audiencias. En cambio, se sugiere en esta investigación el pensar que los medios son los que preparan, como afirma Luhmann, ${ }^{3}$ el trasfondo y prescriben el marco a partir del cual ellos determinan lo que merece ser comunicado a las audiencias. De este modo, los medios construyen una realidad otra que bien puede ser denominada como "virtual".

El desarrollo de este texto pasa también por la constatación de que las iglesias en México todavía no logran aprovechar el potencial de difusión y penetración que tienen los medios en la actualidad, de manera muy particular el poder que tiene la radio y la televisión para difundir mensajes, y poder ofrecer sus servicios y dar cuenta a los demás sobre su trabajo pastoral. Mucho del trabajo evangelizador de las iglesias, particularmente de la Iglesia católica, se centra en el uso de medios de muy corto alcance y de bajo costo (boletines, reproducción de canciones, proyección de diapositivas, videos y películas), pero no se ha explotado, hasta ahora, suficientemente el potencial de difusión de los medios de masas (revistas, periódicos, radio, televisión y últimamente Internet, que se ha convertido en una especie de ágora electrónica) desde donde las iglesias bien pueden hacer, como se hace en otros países, su labor de proselitismo y evangelización.

Hay que decir, sin embargo, en descargo de las iglesias en nuestro país, que la Ley de Asociaciones Religiosas y Culto Público en México, emitida en 1992, deja sin demasiadas posibilidades a las iglesias para administrar y operar sus propios medios de comunicación electrónicos. Esta ley, en su artículo 16, señala que:

Las asociaciones religiosas y los ministros de culto no podrán poseer o administrar, por sí o por sí o por interpósita persona concesiones para la explotación de estaciones de radio, televisión o cualquier tipo de telecomunicación, ni adquirir, poseer o

3. Niklas Luhmann: La realidad de los medios, capítulo 9 (mimeo). 
administrar cualquiera de los medios de comunicación masiva. Se excluyen de la presente prohibición las publicaciones impresas de carácter religioso. ${ }^{4}$

Por lo que toca a la Iglesia católica mexicana, ésta tiene una concepción muy romántica del papel de los medios, pues los concibe como promotores de la comunión entre los seres humanos, lo cual, como se sabe, a partir de la experiencia práctica casi nunca sucede y los medios son en realidad empresas lucrativas que explotan las condiciones económicas, culturales y el amplio desarrollo tecnológico en favor de acrecentar su poder político, económico y social, olvidándose de la potencial importancia que tendrían todos estos medios como vehiculadores de las diversas experiencias comunitarias y de servicio social y como agentes indispensables en la promoción del cambio.

Habrá que decir, en este sentido, que los medios más bien han sido transmisores de una visión falseada de la vida, de las relaciones sociales, de los valores familiares y de la religión misma. Por otro lado, los medios no han observado el suficiente respeto por la privacidad de la vida y en un afán por ganar la noticia han violentado momentos de intimidad poniendo por encima de cualquier consideración el espectáculo público o el acrecentar su rating. Por este camino, en nuestro entorno, los medios se han convertido en tremendos jueces y al hacerlo, algunas veces, le han robado la honra a la gente y después, cuando se descubre que se han equivocado, ¿cómo se la devuelven? Por otra parte, al convertirse en jueces, los medios se otorgan una capacidad única de violar la intimidad de la vida de las personas, lo cual es verdaderamente aterrador e intolerable aun en aras del bien y del servicio social que los medios deberían de prestar.

Este tipo de situaciones, donde los medios han violentado e invadido el espacio de lo privado, han llevado a algunos grupos a protestar y a buscar la manera de impulsar iniciativas concretas que buscan tutelar el derecho de los particulares a la privacidad y el respeto de su intimidad y honor, buscando con ello poner coto a la intromisión de los medios en su afán de conseguir la noticia. ${ }^{5}$

Por lo que toca a los propietarios de los medios electrónicos, éstos tampoco han mostrado demasiado interés en hacer de los asuntos religiosos materia de difusión en sus diversos espacios noticiosos. Apenas en el noticiero nocturno de los domingos, Televisión Azteca da a conocer una nota sobre la misa del cardenal Rivera y el Canal 9 de Televisa transmite las mañanas de domingo (9:00 am) una misa desde la Basílica de Guadalupe.

Si bien es cierto (y esto se relaciona con la primera hipótesis) que con la proximidad de la cuarta visita de! Pañ ? ........ (lescera y cuarta hipótesis) las

4. Ley de Asociaciones Religiosas y Culto Público, artículo 16.

5. Una de las iniciativas de este tipo se dio en Colombia a partir del papel jugado por los medios en la entrega, por parte de la guerrilla, de los soldados prisioneros de guerra. 
noticias respecto a los asuntos religiosos se han incrementado notablemente. Incluso, se están presentando una serie de promocionales sobre la visita del prelado católico.

Es importante destacar, sin embargo, que en la televisión por cable se ha estructurado un canal dedicado a ventilar diversos asuntos religiosos (Claravisión, el cual opera desde la ciudad de Toluca). No hay que olvidar, por una parte, que la televisión por cable no está reglamentada suficientemente en nuestro país, de modo que es más fácil lograr los permisos para operar una televisora de estas características y, por otra parte, la televisión por cable tiene aún poca penetración entre nosotros, de manera que puede ser considerada como de muy bajo impacto, a diferencia de lo que pasa en países como Estados Unidos, ${ }^{7}$ donde la mayoría de las iglesias se han apropiado y están haciendo uso de todos estos recursos para difundir sus mensajes, sus servicios, afiliar a más feligreses y obtener fondos para llevar adelante sus distintos programas de trabajo.

Dentro de la Iglesia católica, el mismo Juan Pablo II ha asumido que los medios de comunicación son un reto para la misión evangelizadora de la Iglesia, y al respecto ha escrito en una de sus varias encíclicas:

el mensaje evangélico deberá, sí, llegar a través de ellos, a las muchedumbres, pero con capacidad para penetrar en las conciencias, para posarse en el corazón de cada hombre en particular, con todo lo que éste tiene de singular y personal y con capacidad para suscitar un favor suyo una adhesión y un compromiso verdaderamente personales. 8

En cuanto a la Iglesia católica mexicana, ésta no termina de definir un proyecto de trabajo claro, preciso e inteligente con respecto al uso de los medios de comunicación y su potencial de difusión pastoral, si bien el diagnóstico hecho por la Conferencia del Episcopado Mexicano (CEM) es acertado cuando afirma que:

Constatamos que hay poca presencia de la iglesia en los grandes medios de comunicación. No hemos sabido evangelizar los medios de comunicación ni hemos desarrollado una adecuada pastoral a través de ellos, para el servicio del evangelio. ${ }^{9}$

6. Destaca la serie de reportajes que Televisión Azteca estructuró en torno a la cuarta visita del Papa a México. Estos reportajes iniciaron la noche del primero de noviembre de 1998. De igual forma, CNI, Canal 40 (hoy Azteca 40) transmitió el lunes 2 de noviembre de 1998 un reportaje sobre "Iglesias electrónicas".

7. Es notoria la experiencia de la cadena norteamericana TBN, la cual en frecuencia abierta transmite diversos programas religiosos desde Santa Ana, California.

8. Evangelii Nuntiandi, 45.

9. Plan Pastoral CEM 1996-2000, numeral 131. 
No por ello se ha dado una estrategia que tienda a resolver el problema o por lo menos a revertirlo buscando hacer de los medios "aliados" privilegiados de su labor pastoral y evangélica: "proclamar la buena nueva a todos los rincones del orbe".

Más bien, el Episcopado mexicano ha manifestado una confianza desmedida en que han de ser los laicos quienes asuman la responsabilidad y capacidad de permear los medios, dejando que sean los propietarios de los medios quienes abran diversos espacios para la difusión de los servicios religiosos. En la práctica, esta esperanza no sólo es ilusoria, sino que además es sumamente endeble y complicada de llevar a cabo, pues los criterios de operación de los medios no son pastorales sino financieros; los medios son empresas de lucro, y más valdría que lo entenderían así los grupos religiosos. El lema de operación de los medios se puede resumir con la siguiente frase: "a mayor rating mayor rentabilidad y mayores ganancias".

La práctica diaria muestra que los medios, cuando se interesan por los fenómenos religiosos, lo hacen atraídos por aquellos sucesos o personajes que revistan espectacularidad y que tienen una capacidad real de convocatoria, tal es el caso de los constantes viajes del Papa, pues Juan Pablo II se ha convertido en un buen actor, capaz de aglutinar en torno a su figura a muchos millones de "tele-espectadores". Juan Pablo II, o mejor dicho, la instancia papal, es sin duda una figura de muy alto rating, y por ese hecho resulta sumamente atractivo para los medios. No es nada gratuito que Televisa haya asignado desde hace ya mucho tiempo a una reportera en el Vaticano.

Siendo así, las iglesias deberían ensayar e imaginar nuevos mecanismos y cauces para poder estar presentes de manera activa en los medios, lo cual no significa abandonar el modo tradicional de servir a los feligreses. La estrategia de estar presentes en y a través de los medios puede y deberá ser múltiple. Así por ejemplo, se puede trabajar en plano legal para modificar la ley con respecto a las Asociaciones Religiosas y Culto Público; se puede trabajar en la profesionalización de los cuadros eclesiásticos para que hagan un uso inteligente y propositivo de los medios; se debe trabajar con los empresarios de medios para conseguir que abran espacios de servicio público que permitan a las distintas iglesias vehicular sus mensajes pastorales y de servicio comunitario; se debe trabajar con los feligreses para pensar mecanismos concretos de operacionalización de sus propios medios así como de un trabajo de educación para un mejor uso de los medios comerciales.

En nuestros días pensar en un trabajo de evangelización a través de los medios ya no resulta una situación extraña, sobre todo si se admite, nos guste o no, que los medios electrónicos tienen una muy amplia presencia física y un notable poder de difusión entre la mayoría de la población. Las cifras que se presentan a continuación reflejan la importancia real que los medios de comuni- 
cación tienen entre nosotros. Se sabe que 96.78 por ciento de las casas tienen aparatos de radio y estima en 2.6 radios por hogar; por otro lado, existen 1332 emisoras de radio en todo el país. También se sabe que existen 507 estaciones televisoras (entre los nueve canales nacionales, repetidoras, televisoras privada locales y televisoras de los gobiernos estatales) De este número, 306 pertenecen a la empresa Televisa, lo que equivale a 50.3 por ciento, 180 a Televisión Azteca lo que constituye 29.7 por ciento, 92 a los gobiernos estatales, es decir, 15.2 por ciento del total y 92 emisoras pertenecen a otro tipo de organismos como el Canal 11 del Instituto Politécnico Nacional (IPN), el Canal 22 del Consejo Nacional para la Cultura y las Artes y el Canal 40 de CNI. ${ }^{10}$

En cuanto a la televisión de paga, existen 213 sistemas de operación de televisión por cable, ocho de televisión por microondas (MDDS) y dos sistemas (Sky y Direct TV) que operan vía satélite y son de reciente ingreso en nuestro país.

Por lo que toca a la prensa escrita, existen en nuestro país 400 periódicos, la mayoría de circulación local; quizá sólo se pueda hablar de unos trece periódicos de circulación nacional, incluyendo al Diario Oficial de la Federación. En cuanto a las revistas, se afirma que hay 192 en nuestro país (casi todas de circulación nacional, de periodicidad variable y que cubren diversas temáticas); también operan 59 agencias de noticias, de las cuales, catorce son nacionales y 45 internacionales; de estas 45, 19 son de Estados Unidos; además hasta el año pasado estaban acreditados en nuestro país 64 corresponsales extranjeros, de los cuales 22 eran de EU. ${ }^{11}$

Con todo este equipamiento es razonable pensar que las iglesias deberían buscar modos concretos de utilizar estos medios para llevar a cabo parte de su trabajo evangelizador.

Es importante destacar que en nuestro país se inició un nuevo capítulo en la relación del Estado con los diversos grupos religiosos a partir de $1992 .{ }^{12} \mathrm{El}$ punto de partida de este proceso de apertura oficial fue la modernización del país, la cual incluye la constitución de un Estado laico moderno, por ende no confesional, y en la práctica ha permitido un importante incremento de los grupos religiosos en el país. Así, por ejemplo, hasta septiembre de 1997 habían sido

10. Canal 40 actualmente forma parte de Televisión Azteca (nota de los editores).

11. Todos los datos antes mencionados fueron tomados del documento Información relevante sobre los medios de Comunicación en México, preparado por la Cámara de Diputados, a propósito de la Conferencia Internacional "El derecho de la información en el Marco de la Reforma del Estado en México", celebrada en la ciudad de México, en mayo de 1998.

12. El marco normativo vigente en materia religiosa en México tiene como punto de partida el decreto publicado en el Diario Oficial de la Federación el 28 de enero de 1992. Mediante este decreto se reformaron los artículos $3^{\circ}, 5^{\circ}, 24,27$ y 130 y se adicionó el artículo 17 transitorio de la Constitución Política de nuestro país. 
registradas en la Secretaría de Gobernación 5,056 asociaciones religiosas, y estaban en trámite 234. Destaca el hecho de que es el Distrito Federal la entidad federativa que más grupos religiosos concentran, con 883 , siendo Aguascalientes, Tlaxcala, Colima, Quintana Roo y Baja California Sur los que menos concentran, con $19,15,12,10$ y 10 asociaciones religiosas, respectivamente. ${ }^{13}$

Con todo y esta apertura y el natural incremento de las asociaciones religiosas y de su consecuente libertad de culto, son todavía muy pocas las iglesias que tienen u operan sus propios medios de comunicación:

Periódicos, radios, televisoras, revistas, páginas de Internet y también se hace poco uso, por parte de las iglesias, de los medios masivos de comunicación como vehiculadores de sus valores, propuestas y servicios religiosos. La Secretaría de Gobernación, por medio de la Dirección General de Asuntos Religiosos, reportó que en 1997 fueron autorizados para su transmisión extraordinaria a través de los medios de comunicación, 987 actos religiosos de culto público. ${ }^{14}$

Este dato aunque en sí mismo puede parecer importante, y de hecho lo es, revela, sin embargo, que los medios de comunicación y las iglesias se han ocupado muy poco por generar una cultura de "servicios religiosos vía los medios masivos de comunicación" y por abrir espacios de encuentros que le permitan entrar, sobre todo a las iglesias, en un nuevo momento de la difusión de sus ideas y servicios religiosos. De suyo, y por lo general en los espacios noticiosos, los medios (radio, prensa y televisión) se han ocupado más bien de asuntos muy específicos como los frecuentes viajes del Papa, los cuales Televisa ha cubierto con amplio detalle. Otro asunto que ocupó los espacios noticiosos fue el cardenalato de Norberto Rivera, el papel de Samuel Ruiz, obispo de San Cristóbal de las Casas, en el conflicto chiapaneco, las declaraciones del cardenal Sandoval Íniguez respecto a la telenovela "Tentaciones" o sus dudas sobre las averiguaciones en torno al asesinato del cardenal Posadas.

Estos datos nos permiten inferir que ni las iglesias se han preocupado suficiente e inteligentemente por explotar el potencial multiplicador de los medios para difundir a través de ellos sus mensajes y servicios, ni los medios se han ocupado seriamente de los asuntos religiosos estableciendo una agenda permanente que dé seguimiento a los diversos acontecimientos relativos a las iglesias y a las prácticas religiosas y destinando para tal fin a personal realmente capacitado para abordar y dilucidar sobre la complicación de los asuntos religiosos.

Quizá habrá que admitir que son las iglesias quienes deberán tomar la iniciativa para constituir este nuevo modelo de difusión de los asuntos religiosos, por razón de su interés y de cumplir con su misión en escenarios nuevos.

13. Los datos han sido tomados de Revista y Sociedad, número 1, Secretaría de Gobernación, octubre-diciembre de 1997, México, páginas 74-88.

14. Ibid., Gráfica 10, página 83 (el dato comprende hasta el 30 de septiembre de 1997). 
Las iglesias deberán aprender que en la actualidad los medios de comunicación tienen un rol muy especial dentro de la conformación de la realidad y son los medios quienes nos dicen (su versión, la única que tiene un carácter suficientemente masificado para ser utilizable como indicador real del acontecer) de lo que está pasando en el mundo; ellos, como afirman los expertos, nos dan su propia versión de la realidad y es esta versión la que se estandariza y es la que terminamos por admitir como realidad. ${ }^{15}$

Se puede discutir si se quiere la calidad, objetividad y veracidad de la información, si lo que los medios nos entregan como información son en realidad medias verdades o artificios para difundir la ignorancia disfrazada de novedad, bajo un maquillaje dramático y escenográfico entretenido que pretende hacernos creer que los medios, y particularmente la televisión, nos hacen ser "tele-testigos" de los acontecimientos diarios y además lo hacen de manera amena.

Quizá se debe advertir a tiempo, para evitar equívocos, que entre el acontecer real, "la realidad real" que llaman algunos, y la producción noticiosa hay una serie de mediaciones, incluyendo las de orden meramente tecnológico las cuales son propias del modo de operar y reconstruir la realidad por parte de los medios. Entre las mediaciones más importantes destacan la dramatización de los formatos noticiosos a través del dramatismo de la música, los avances noticiosos en que se destacan unas noticias frente a otras, la escenografía, el tipo de los conductores que salen cuadro, etc. En la práctica, todos estos elementos hacen que la presentación noticiosa se vaya diluyendo y se presente las noticias como hechos distantes y consumados frente a los cuales la audiencia poco o nada puede hacer.

Es justamente en esta recreación escenográfica en donde se da una de las formas de manipulación más sutiles por parte de los medios, quienes por otra parte se empeñan en hacernos creer que han podido redimir la distancia entre los hechos reales y su presentación massmediática haciendo coincidir el tiempo de los acontecimientos reales con el de su presentación y observación, gracias al notable desarrollo de las tecnologías de información. Este mecanismo se inició (conviene no olvidarlo) con la transmisión que la cadena norteamericana CNN hizo de la guerra del Golfo Pérsico.

15. Luhmann afirma que "Todo lo que sabemos sobre la sociedad y aun lo que sabemos sobre el mundo, lo advertimos a través de los medios de comunicación". La realidad de los medios, (mimeo, página 1). 


\section{Las cuatro hipótesis básicas de esta investigación}

Lo que resta del ensayo lo voy a dedicar a tratar de argumentar sobre cuatro hipótesis de trabajo respecto a la agenda que los medios de comunicación establecen en relación con los asuntos religiosos. Quiero decir, antes de entrar en estas conjeturas, que una de las premisas del proyecto de investigación es el entender que la crítica a los medios pasa por el derecho de tener información confiable.

La primera de las hipótesis conjetura sobre el hecho de que los medios de comunicación se ocupan de los asuntos religiosos como si fueran asuntos que deben ser permanentemente renovados y cuya emergencia en los medios obedece a circunstancias más bien coyunturales. A la base de esta hipótesis existe una constatación empírica: las noticias no pueden dejarse envejecer, pues si se añejan, pierden su valor noticioso, su actualidad y vigencia y con ello el medio pierde credibilidad, pierde rating, es decir, posibilidades de hacer negocios con los anunciantes.

Por esta razón es que vemos que de un día para otro puede desaparecer la continuidad de los asuntos noticiosos; es decir, que se abordan los acontecimientos en la medida en la cual, según el modo de operar de los medios, estos acontecimientos tienen interés general y actualidad.

Así pasa con todo, los asuntos religiosos no son la excepción y son atendidos por los medios, incorporados como parte de su agenda, en la medida en que un hecho que está relacionado con alguna iglesia se convierte, según sus perspectivas, en noticia; es decir, que tiene un valor específico de carácter informativo para el público de ese medio. ${ }^{16}$

Por otra parte, al ser las noticias asuntos que deberán ser permanentemente renovados en aras de mantener a la población "oportunamente" informada, sólo se dará seguimiento a los hechos en la media en que la operacionalización de los medios considere que vale la pena mantener determinada noticia dentro de la agenda, la cual dicho sea de paso funciona sobre la base de una selectividad continua centrada en los intereses de los medios y no de las audiencias o de la importancia de los acontecimientos mismos.

Las noticias consideradas por los medios como irrelevantes pierden su valor y su vigencia y pasan a engrosar los archivos de los bancos saturados de información, y de los cuales después se podrán rescatar algunas notas que podrían servir de material de archivo para ambientar y estructurar algunos reportajes especiales.

15. En este mismo texto Luhmann afirma que: "La información no se presta a la repetición. Una

4 noticia que es emitida más de una vez, sigue conservando sentido, pero pierde valor de información", página 16. 
En descargo de los medios habrá de decirse que las audiencias acostumbradas y educadas en la vertiginosidad ya no aceptamos tan fácilmente la repetición de las noticias, pues también hemos aprendido a valorar que la información debe ser nueva para que tenga valor ${ }^{17}$ En la práctica, las cosas suceden así hasta el punto de que la agenda de los medios crea la necesidad de sustituir la información que ya se ha vuelto redundante, que ha perdido su valor de cambio, por una información fresca que tiene el atractivo de la novedad como su valor máximo.

Hay pues, en la dinámica noticiosa de los medios, una especie de permanente actualización/desactualización de la información o, lo que es lo mismo, se hace una valoración entre lo novedoso y lo obsoleto en términos informativos y es éste el criterio que priva a la hora de presentar el acontecer noticioso como si fueran los hechos del mundo.

En la investigación realizada, los casos que ejemplifican este tratamiento de las noticias, donde los medios operan con el criterio de la sorpresa y la estandarización, a la vez, como pilares que producen el valor noticioso, abundan tanto a nivel nacional como internacional. En el momento en que se comenzó a redactar este texto, ya se comenzaba a tratar el asunto de la cuarta visita del Papa a México, en enero de 1999. Lo que inició esta temática noticiosa fue el hecho de que estuvo en México por esos días - febrero de 1998- el coordinador de los viajes del Papa, quien prometió volver antes de terminar el año para ultimar los detalles del cuarto viaje del pontífice a nuestro país. ${ }^{18}$ Para el mes de octubre, comenzaron a aparecer los promocionales de la visita del pontífice y se dio difusión en los medios al vídeo de la canción oficial para la visita del jerarca católico; por otra parte, se comenzaron a hacer algunos reportajes sobre el Papa y su relación con México.

Este modo de vértigo permanente con que operan los medios, se puede ver también en el caso de la visita del Papa a Cuba, en enero de 1998. Unas semanas después se dejó de hablar de esa visita, y los impactos que entre la población cubana tuvo aquel viaje que en su momento generó mucha información. De igual modo, ya no se habla del asesinato del obispo Gerardi en Guatemala, y ni siquiera de la recepción del Papa al futbolista brasileño Ronaldo o a los integrantes de la selección mexicana. Hasta el obispo Samuel Ruiz, actor fundamental dentro del proceso de pacificación en Chiapas, ha tenido que pagar el precio de la lógica con que operan los medios basados en la necesidad permanente de la actualidad y novedad noticiosa.

17. Como se sabe, algo muy distante ocurre con la publicidad, la cual opera sobre la base de la repetición, del posicionamiento y de la reiteración continua, buscando que las audiencias no olviden, a la hora de hacer sus compras, las potenciales virtudes de los productos publicitados.

18. La revisión de las noticias, en ese momento, indicaba que del mismo modo como se habló del asunto un par de días, desapareció de la agenda y no volvió a hablarse del tema hasta cuando se tuvo un nuevo elemento en torno al asunto. 
Vemos que todos y cada uno de los diversos actores van y vienen dentro de la pantalla y las páginas de los periódicos, y que hay veces en que son desplazados por otras noticias que llegan un día para conformar la agenda y el acontecer noticioso, siempre guiado por la necesidad y la novedad, y de la primicia noticiosa para ganarle audiencia a la competencia. Así, por ejemplo, recientemente se dio cuenta en la prensa de las rogativas a la Virgen de Guadalupe en San Luis Potosí para que lloviera en ese estado; también por esos mismos días salió a relucir la figura del obispo Samuel Ruiz haciendo cuerpo con los demás obispos de Chiapas para desmentir que la Iglesia católica en aquel estado tenga y difunda una "Teología de la Violencia". Al día siguiente los medios nos presentaron otras noticias, otros aconteceres, sin continuidad posible y así vamos, como audiencias, siendo guiados por la fuerza de la coyuntura y de la novedad noticiosa. Por ejemplo, a estas alturas, ya nadie se ocupa en los medios de la imagen de la virgen aparecida en el metro Hidalgo.

Estos ejemplos sirven para afirmar que en los medios lo que ya pasó es "historia", parte de los oldtimer, y ellos no se ocupan de lo histórico sino de lo actual, de lo nuevo dentro del diario acontecer; es decir, de lo que tiene un valor informativo, un valor de venta en el mercado noticioso donde nada permanece para siempre.

La segunda hipótesis con la que se trabajó en el proyecto de investigación es que los medios no tienen, ni les interesa tener, expertos que se encarguen de dar un tratamiento serio a los asuntos religiosos (a diferencia de otros aspectos como la política, la economía y los deportes) y siendo así, en realidad lo que difunden es ignorancia respecto a este complicado asunto.

Esta hipótesis parte de la premisa de que, en general, los medios tienen muy poco trabajo editorial, incluso la prensa escrita que es donde más se editorializa, la mayor parte de su agenda respecto a los asuntos religiosos es de tipo noticioso y no de artículos de opinión ni de análisis, y cuando los hay son para atacar, desacreditar y deslegitimar; muy pocas veces para orientar al lector o al receptor para que éste se forme una opinión más precisa de la problemática.

En la investigación se ha descubierto que sobre los temas religiosos se editorializa muy poco en la prensa nacional. Incluso periódicos como La Jornada y El Financiero, publicaciones que tienen importantes secciones de artículos de opinión, poco opinan sobre este fenómeno, y la mayor parte de su cobertura sobre asuntos religiosos es de tipo noticioso o se trata de reportajes. Esto hace pensar que en los medios no hay expertos que sean capaces de abordar la complejidad de estos asuntos para dar una opinión inteligente y sensata sobre el acontecer religioso en nuestro país. De esa manera, por lo general termina repitiendo lugares comunes y haciendo refritos de noticias para llenar los espacios, y cuando alguien se mete a opinar sobre los asuntos religiosos lo hace con una simpleza y vaguedad que dan escalofríos. 
Por ejemplo, en el caso del problema religioso en Chiapas (que tiene muchas aristas, pues se relaciona con lo étnico, con lo político, con la historia, con la presencia y participación de diversos grupos religiosos, con la educación y las condiciones de marginalidad del estado, etc.) las noticias no hacen un abordaje integral que refleje la complejidad del momento que se vive en esa entidad y la complejidad histórica del problema indígena en nuestro país, y dentro de esa complejidad el papel que los grupos y figuras religiosos juegan en esa entidad. Más bien, lo que vemos o leemos en la prensa es una confusión y un entrecruzamiento de hilos noticiosos que hacen difícil el seguir la argumentación y las tramas noticiosas.

Esta ambigüedad sobre lo religioso es ilustrativa del modo en que los medios abordan temáticas en las cuales no tienen expertos capaces de dilucidar sobre la complejidad del fenómeno y las interrelaciones que este tema tiene respecto del resto del acontecer de una comunidad. El caso de Chiapas es ilustrativo, pues en ese lugar hay una concurrencia de actores y factores (incluyendo lo religioso) que hacen delicada y peligrosa la situación y lo que los medios nos informan hace manifiesto que ellos no logran atender esa complejidad de manera seria y profunda.

La tercera hipótesis conjetura sobre la idea de que los medios, al igual que en otros ámbitos noticiosos, se ocupan de lo religioso atendiendo principalmente a poner de manifiesto las opiniones de las figuras de autoridad de las iglesias; de los jerarcas de las iglesias y no de la feligresía.

Una variante de esa situación se da a través de lo que algunos han denominado la exteriorización de opiniones, es decir, la entrevista a expertos cuyas opiniones son consideradas por los medios como noticias. ${ }^{19}$ En el campo de lo religioso, para lograr su cometido los medios suelen consultar a los jerarcas, considerándolos como figuras centrales del campo de lo religioso; junto a esto, los medios algunas veces también consultan a los contados "expertos" en esta materia para que viertan sus opiniones, y con ello los medios llenan sus espacios de críticas y comentarios diversos, dejando que sean estas figuras de autoridad quienes acentúen su propia versión de los hechos.

Este culto a la personalidad, esta búsqueda de los medios por las figuras de autoridad no es nada nueva y se da en todos los ámbitos del acontecer noticioso. Los medios recurren en busca de los informantes cualificados (ésos que tienen poder y juegan un rol dentro de la estructura social y en el caso que nos ocupa dentro de la estructura religiosa). De modo que, al igual que en la política, en los deportes, en lo social y cultural hay una centralidad básica de las figuras de autoridad estableciéndose así, un culto a la personalidad muy manifiesta.

19. Ver el libro La realidad de los medios de Niklas Luhmann, en especial el punto 9 de su análisis de las noticias. 
No es de extrañar, entonces, que quienes están en el centro de las noticias religiosas sean en ese orden: el Papa, el cardenal Norberto Rivera, el embajador Justo Mullor, el cardenal de Guadalajara, el obispo Samuel Ruiz, y de vez en cuando algunos miembros de la Conferencia Episcopal Mexicana (CEM), pero nunca o casi nunca los párrocos y mucho menos los laicos y feligreses, salvo, iclaro!, cuando se trata de reportar casos específicos de la cultura religiosa como las celebraciones de Semana Santa, pero aun allí quienes ocupan los espacios de los medios son las figuras protagónicas (el que la hizo de Jesús, la que la hizo de María Magdalena y por supuesto estas noticias se generan en el espacio de la celebración de la Semana Santa — lo adivinó usted-, en Iztapalapa).

La estrategia de los medios, pues, busca hacer referencia a la reputación notable de la fuente consultada y de este modo crea su propia agenda, acentuando lo que a juicio de los medios debe ser objeto de opinión y de noticia.

Es muy posible que para efectos propios de la investigación, esta hipótesis debe cruzarse con las estrategias y equipos de trabajo que para con los medios han establecido algunos de estos personajes de la cúpula eclesiástica. Es muy posible que una estrategia bien planeada de trabajo y de atención a los medios convierta a cada uno de estos personajes en protagonista del acontecer noticioso que se agenda y estructura en los medios de comunicación, y que justamente esta circunstancia de atención y trabajo con los medios sea lo que haga la diferencia entre personajes del mismo rango como podría ser el caso del cardenal de Monterrey, quien tiene muy poca presencia en los medios, según algunos debido a su precario estado de salud, pero sobre todo a su mala relación con los medios.

La cuarta hipótesis se refiere al hecho de que la mayoría de noticias acopiadas durante estos meses de investigación en la prensa mexicana, están relacionadas directa y fundamentalmente con acontecimientos vinculados a la Iglesia católica, hechos en los que participan miembros de la jerarquía católica o, en el mejor de los casos, se reportan prácticas religiosas que tienen que ver con prácticas religiosas de contenido católico. Hay una altísima cantidad de noticias que se ocupan de dar cuenta de la actividad u opiniones de los jerarcas de la Iglesia católica, tanto en el ámbito nacional como internacional. ${ }^{20}$

La revisión de las noticias hechas durante el año pasado revela que muy pocas noticias se ocupan de los asuntos relacionados con otras iglesias y grupos religiosos no católicos; esto sucede tanto en el ámbito nacional como internacional. Tanto es así que las opiniones de los jerarcas de las demás iglesias así como los rituales y prácticas religiosas de sus feligreses parecen no existir en la agenda actual de los medios, particularmente de la prensa en México.

20. Destaca en este sentido la opinión y el disgusto que provocó en el Vaticano la designación de José Saramago como ganador del premio Nobel de Literatura 1998. 
Este predominio de lo católico, como tema noticioso, revela, por un lado, que en efecto en nuestro país lo católico tiene una predominancia real dentro del campo de lo religioso, y que los grupos no católicos continúan siendo todavía una minoría. ${ }^{21}$ Por otro lado, también revela el modo de proceder y conformar la agenda noticiosa por parte de los mismos medios, quienes ponderan más a los grupos de poder constituidos y no a los grupos sociales emergentes, como podrían ser nuevos movimientos religiosos, como la Iglesia de la Unificación del reverendo Moon; el grupo Portal del Cielo, el cual se puso de moda por el suicidio colectivo de varios de sus miembros en marzo de 1997 en la ciudad de San Diego, California; los davidianos, seguidores de David Koresh, muerto en Waco, Texas; el New Age; los tele-evangelistas liderados por Morris Cerullo, quien en marzo de 1997 estuvo en la ciudad de México y abarrotó el Zócalo capitalino y otros tipos de iglesias de corte orientalista.

Además de esos nuevos movimientos religiosos, los medios han dejado fuera de su agenda noticiosa a otros grupos, como las sectas e iglesias no católicas ya constituidas, como: la iglesia La Luz del Mundo, los grupos pentecostales, los fundamentalistas, los mormones, los testigos de Jehová y, por supuesto, poco o nada se dice de las iglesias históricas: anglicanos, luteranos, calvinistas y los disidentes de ese tipo de iglesias como son los metodistas, los bautistas, puritanos y otros grupos religiosos, como los judíos, los musulmanes, etcétera.

21. Un texto interesante que revela los actuales alcances del fenómeno no católico en América Latina es el libro Secta o Iglesias: Viejos o nuevos movimientos religiosos. Compilado por Elio Maferre, editado por Aler-Plaza y Valdés, México, 1998. 